\title{
The Effect of the Primitive Irreducible Polynomial on the Quality of Cryptographic Properties of Block Ciphers
}

\author{
Sajjad Shaukat Jamal $\mathbb{D}^{1},{ }^{1}$ Dawood Shah, ${ }^{2}$ Abdulaziz Deajim, ${ }^{1}$ and Tariq Shah ${ }^{2}$ \\ ${ }^{1}$ Department of Mathematics, College of Science, King Khalid University, P. O. Box 9004, Abha, Saudi Arabia \\ ${ }^{2}$ Department of Mathematics, Quaid-i-Azam University, Islamabad, Pakistan \\ Correspondence should be addressed to Sajjad Shaukat Jamal; shussain@kku.edu.sa
}

Received 23 May 2020; Revised 3 August 2020; Accepted 28 August 2020; Published 24 September 2020

Academic Editor: Tom Chen

Copyright (C) 2020 Sajjad Shaukat Jamal et al. This is an open access article distributed under the Creative Commons Attribution License, which permits unrestricted use, distribution, and reproduction in any medium, provided the original work is properly cited.

Substitution boxes are the only nonlinear component of the symmetric key cryptography and play a key role in the cryptosystem. In block ciphers, the S-boxes create confusion and add valuable strength. The majority of the substitution boxes algorithms focus on bijective Boolean functions and primitive irreducible polynomial that generates the Galois field. For binary field F2, there are exactly 16 primitive irreducible polynomials of degree 8 and it prompts us to construct 16 Galois field extensions of order 256 . Conventionally, construction of affine power affine S-box is based on Galois field of order 256, depending on a single degree 8 primitive irreducible polynomial over $\mathbb{Z}_{2}$. In this manuscript, we study affine power affine S-boxes for all the 16 distinct degree 8 primitive irreducible polynomials over $\mathbb{Z}_{2}$ to propose 16 different $8 \times 8$ substitution boxes. To perform this idea, we introduce 16 affine power affine transformations and, for fixed parameters, we obtained 16 distinct S-boxes. Here, we thoroughly study S-boxes with all possible primitive irreducible polynomials and their algebraic properties. All of these boxes are evaluated with the help of nonlinearity test, strict avalanche criterion, bit independent criterion, and linear and differential approximation probability analyses to measure the algebraic and statistical strength of the proposed substitution boxes. Majority logic criterion results indicate that the proposed substitution boxes are well suited for the techniques of secure communication.

\section{Introduction}

The exchange of digital data through the Internet has revolutionized the communication parameters over the years. But this rapid communication also provides opportunities to access this digital data illegally. For this reason, the security of this content on the Internet has become a serious challenge for the researchers of different fields. To counter the emerging challenges of security, cryptography and steganography are used to hide the secret information whereas watermarking is used for copyright protection. In this manuscript, we discuss cryptography and relevant aspects of this field. For convenience, cryptography is divided into two types named symmetric key cryptography and asymmetric key cryptography. In symmetric key cryptography, two parties share secret information and keys during encryption and decryption procedures. The private key is shared by both sender and receiver. In addition to this, block ciphers and stream ciphers are two main branches of symmetric key cryptography. In 1949, Shannon gave the idea of block cipher and some examples of block ciphers are Advanced Encryption Standard (AES) [1], Data Encryption Standard (DES), International Data Encryption Algorithm (IDEA), and many more [2,3]. In AES, there is availability of three different key sizes such as 128,192 , and 256 bits, whereas in DES, the only available key size is 56 bits. The AES has 10, 12, and 14 rounds for key sizes of 128, 192, and 256 bits, respectively. All these rounds have four basic steps, that is, subbyte, shift row, mix column, and add round key. Subbyte is the step which substitutes the plaintext data with substitution box (S-box). This S-box is the only nonlinear part of block cipher used in different well-known cryptosystems. It is used to create confusion to make plaintext data obscure for any attacker and hence S-box is an integral part of any cryptosystem. S-box is a function which has input and output from the Galois field. The Galois field is a finite field having order 256 and denoted by $\mathrm{GF}\left(2^{8}\right)$. 
1.1. Related Work. S-box is used to create confusion as observed in AES, International Data Encryption Algorithm (IDEA), DES, and many more cryptosystems [4]. It is an established fact that the strength of block cipher depends on the standard and quality of S-box. Due to the necessary immersion of S-box to generate nonlinearity, intricacy persuades different researchers to design strong S-boxes to enhance the security level of cryptosystems. Among different available methods, the algebraic structure-based construction of S-boxes has much more attention. These S-boxes have strong cryptographic features and are robust against linear and differential cryptanalysis.

In the literature, different structural advancements are viewed to improve the quality of S-boxes. The algebraic complexity of AES S-box has been improved with the extension of this S-box, that is, affine power affine (APA) [5]. Furthermore, the symmetric group $S_{8}$ has also been applied to AES S-box to improve the quality and numbers of S-boxes [6]. Similarly, the application of transformation using binary gray codes on AES S-box gives Gray S-box [7]. In [8], S-boxes are constructed by using the projective general linear group (PGL). Moreover, the construction scheme of chaotic S-boxes using DNA sequence and chaotic Chen system is given in $[9,10]$. Different analytical, algebraic, and chaos-based techniques for the construction of S-boxes are given in [11-16]. Conventionally, AES uses a polynomial of 8 terms which have all the required properties and improves the security for AES. But the Gray S-box has a 255-term polynomial. Moreover, residue prime, Xyi, and Skipjack S-boxes are frequently used for the encryption and decryption schemes $[17,18]$.

It is assumed that the model of Boolean functions and primitive irreducible polynomial has an impact on the strength of S-box. In [19], different primitive irreducible polynomials have been used to identify the effect of primitive irreducible polynomial. To investigate this fact, we want to study all the primitive irreducible polynomials to understand whether there is an impact of irreducible polynomial or not. Archetypally in the synthesis of an S-box, the numbers $a, b, c$, and $d$ in affine transformation belong to Galois field $\mathrm{GF}\left(2^{8}\right)$. As the polynomial ring $\mathbb{Z}_{2}[x]$ has 16 primitive irreducible polynomials of degree 8 , it shows that only 16 opportunities are available for constructing Galois fields $\mathrm{GF}\left(2^{8}\right)$. In this paper, we have constructed 16 different robust $8 \times 8 \mathrm{~S}$-boxes over the elements of these 16 irreducible polynomials. Firstly, we define 16 affine power affine transformations on these different Galois fields which can be given as $z \longrightarrow(a z+b) o(c z+d)^{-1}$; here, for $a, b, c, d$ values, we would be able to get 16 distinct S-boxes.

1.2. Motivation. Due to the role of S-boxes in cryptosystems, it is essential to explore all of its aspects. The motivation behind this work is to study all primitive irreducible polynomials and their role in the construction of S-boxes.
(1) The Mobius transformation used in a different construction of S-boxes has certain limitations and restrictions in its structure [7]. For example, the condition on the parameters, i.e., $a d-b c \neq 0$ $\forall a, b, c, d \in \mathrm{GF}\left(2^{8}\right)$ squeezes the remaining cases. Hence, there is a need for any other transformation.

(2) There are 16 primitive irreducible polynomials in the principal ideal domain $\mathbb{Z}_{2}[x]$ whose impact was not studied yet regarding their impression on analyses of S-boxes.

(3) By exploring all primitive irreducible polynomials, we have a better opportunity to obtain the cryptographically strong cryptosystems.

1.3. Our Contribution. In this manuscript, we studied all binary degree 8 primitive irreducible polynomials for the construction of S-boxes. The quality of the proposed work can be seen from the different security analyses and resistance against malicious attacks. This whole study can be summarized as follows:

(1) We constructed S-boxes associated with the 16 binary degree 8 primitive irreducible polynomials.

(2) The APA transformation is used in this work, which is bijective and has no restrictions on the parameters.

(3) To evaluate the strength of the proposed S-boxes, we have performed different analyses along with differential cryptanalysis. The outcomes of these analyses are compared with the well-known S-boxes.

The remaining part of the paper is planned as follows: Section 2 presents the preliminaries and construction scheme of the proposed S-boxes. In Section 3, algebraic and statistical analyses are calculated in detail. Section 4 presents definitions of the balanced Boolean function. Section 5 concludes the paper.

\section{Primitive Irreducible Polynomials of Degree 8 and GF $\left(2^{8}\right)$}

2.1. The Galois Fields $G F\left(2^{8}\right)$. We summarize here some well-known facts from the theory of rings and fields. Let $R$ be a commutative ring with identity. A nonempty subset $J$ of $R$ is called an ideal of $R$ if $J$ is an additive subgroup of $R$ and $a J \subseteq J$ for every $a \in R$, where $a J=\{a j \mid j \in J\}$. If, furthermore, there does not exist a proper ideal of $R$ properly containing $J$, then we say that $J$ is a maximal ideal of $R$ Besides; $R$ is said to be a field if each of its nonzero elements has a must inverse in $R$. If $R$ is a field of prime characteristic $p$, then $R$ is an extension of the prime field $\mathbb{Z}_{p}$. A polynomial $f(x) \in \mathbb{Z}_{p}[x]$ is said to be irreducible if it cannot be factored in $\mathbb{Z}_{p}[x]$ into two polynomials of strictly smaller degrees. The principal ideal,

$$
\langle f(x)\rangle=\left\{h(x) f(x): h(x) \in \mathbb{Z}_{p}[x]\right\},
$$


generated by a monic irreducible polynomial $f(x) \in \mathbb{Z}_{p}[x]$ is a maximal ideal in $\mathbb{Z}_{p}[x]$. If $f(x)$ is of degree $m$, then the quotient ring,

$$
\frac{\mathbb{Z}_{p}[x]}{\langle f(x)\rangle}=\left\{g(x)+\langle f(x)\rangle: g(x) \in \mathbb{Z}_{p}[x]\right\},
$$

is an extension field of $\mathbb{Z}_{p}$ of degree $m$ consisting of $p^{m}$ elements. This field is called a Galois field and is denoted by $\mathrm{GF}\left(p^{m}\right)$ and is said to be the field extension of $\mathbb{Z}_{p}$ defined by the irreducible polynomial $f(x)$. A representative $g(x)$ of each element of $\mathrm{GF}\left(p^{m}\right)$ can be chosen to be of degree strictly less than $m$. If $\alpha$ is a root of $f(x)$ in an algebraic closure of $\mathbb{Z}_{p}$, then $\mathrm{GF}\left(p^{m}\right)$ is isomorphic to the field:

$$
\mathbb{Z}_{p}(\alpha)=\left\{a_{0}+a_{1} \alpha+\cdots+a_{m-1} \alpha^{m-1}: a_{i} \in \mathbb{Z}_{p}, \forall i\right\},
$$

and so we can identify the two fields. Furthermore, if $\alpha$ is a generator of the cyclic finite multiplicative group of nonzero elements of $\mathbb{Z}_{p}(\alpha)$, then we say that $f(x)$ is primitive.

The Galois field GF $\left(2^{8}\right)$ is particularly of specific interest in cryptographic applications, especially in S-boxes constructions. For our cryptographic purposes, we are interested in such a field whose defining irreducible polynomial is "primitive" (of degree 8 , of course). It is well known that there are $\left(\varphi\left(2^{8}-1\right) / 8\right)=16$ such polynomials over $\mathbb{Z}_{2}$, for example, $p_{1}(x), \ldots, p_{16}(x) \in \mathbb{Z}_{2}[x]$, which we list in Table 1 . In the following section, we construct 16 S-boxes out of the Galois fields corresponding to the aforementioned sixteen primitive irreducible polynomials.

2.2. The Proposed S-Box Construction Method. For each $i=1, \ldots, 16$, consider the affine power affine map (APA):

$$
S=A_{1} \circ f \circ A_{2}: \mathbb{Z}_{2}\left(\alpha_{i}\right) \longrightarrow \mathbb{Z}_{2}\left(\alpha_{i}\right),
$$

where $\quad A_{1}(x)=a x+b \quad$ and $\quad A_{2}(x)=c x+d$ $\left(a, b, c, d \in \mathbb{Z}_{2}\left(\alpha_{i}\right)\right)$ are two affine maps with $a, c \neq 0$, and

$$
f(x)= \begin{cases}x^{-1}, & \text { if } x \neq 0 \\ 0, & \text { if } x=0 .\end{cases}
$$

Among other things, the map $S$, which is obviously bijective, was introduced by [5] to produce confusion in the scheme. For our S-boxes, we choose $a=13, b=14$ and $c=$ 102 and $d=210$. Figure 1 demonstrates the flow chart of the construction of the 16 different S-boxes. Moreover, the construction of S-boxes in correspondence to polynomial 1 $\left(P_{1}\right)$ to polynomial $16\left(P_{16}\right)$ is shown in Figure 1 . All the S-boxes are given in Tables $2-17$, corresponding to $P_{1}$ to $P_{16}$. These tables are before the conclusion section.

In the proposed work, we present an APA S-box corresponding to each $i=1, \ldots, 16$ where the APA map $S$ gives the $16 \times 16$ lookup tables. We, then, show that these S-boxes have strong cryptographic properties certified with the help of analyses such as nonlinearity, strict avalanche criterion (SAC), bit independent criterion (BIC), linear approximation probability (LP), and differential approximation probability (DP) [20].
TABle 1: Primitive irreducible polynomials and their corresponding Galois fields.

\begin{tabular}{lc}
\hline Primitive polynomials $p_{i}(x) \&$ roots $\alpha_{i}$ & Galois field GF $\left(2^{8}\right)$ \\
\hline$p_{1}(x)=x^{8}+x^{4}+x^{3}+x^{2}+1 ; \alpha_{1}$ & $\mathbb{Z}_{2}[x] /\left\langle p_{1}(x)\right\rangle$ \\
$p_{2}(x)=x^{8}+x^{5}+x^{3}+x+1 ; \alpha_{2}$ & $\mathbb{Z}_{2}[x] /\left\langle p_{2}(x)\right\rangle$ \\
$p_{3}(x)=x^{8}+x^{5}+x^{3}+x^{2}+1 ; \alpha_{3}$ & $\mathbb{Z}_{2}[x] /\left\langle p_{3}(x)\right\rangle$ \\
$p_{4}(x)=x^{8}+x^{6}+x^{3}+x^{2}+1 ; \alpha_{4}$ & $\mathbb{Z}_{2}[x] /\left\langle p_{4}(x)\right\rangle$ \\
$p_{5}(x)=x^{8}+x^{6}+x^{4}+x^{3}+x^{2}+x+1 ; \alpha_{5}$ & $\mathbb{Z}_{2}[x] /\left\langle p_{5}(x)\right\rangle$ \\
$p_{6}(x)=x^{8}+x^{6}+x^{5}+x+1 ; \alpha_{6}$ & $\mathbb{Z}_{2}[x] /\left\langle p_{6}(x)\right\rangle$ \\
$p_{7}(x)=x^{8}+x^{6}+x^{5}+x^{2}+1 ; \alpha_{7}$ & $\mathbb{Z}_{2}[x] /\left\langle p_{7}(x)\right\rangle$ \\
$p_{8}(x)=x^{8}+x^{6}+x^{5}+x^{3}+1 ; \alpha_{8}$ & $\mathbb{Z}_{2}[x] /\left\langle p_{8}(x)\right\rangle$ \\
$p_{9}(x)=x^{8}+x^{7}+x^{3}+x^{2}+1 ; \alpha_{9}$ & $\mathbb{Z}_{2}[x] /\left\langle p_{9}(x)\right\rangle$ \\
$p_{10}(x)=x^{8}+x^{7}+x^{5}+x^{3}+1 ; \alpha_{10}$ & $\mathbb{Z}_{2}[x] /\left\langle p_{10}(x)\right\rangle$ \\
$p_{11}(x)=x^{8}+x^{7}+x^{2}+x+1 ; \alpha_{11}$ & $\mathbb{Z}_{2}[x] /\left\langle p_{11}(x)\right\rangle$ \\
$p_{12}(x)=x^{8}+x^{7}+x^{6}+x+1 ; \alpha_{12}$ & $\mathbb{Z}_{2}[x] /\left\langle p_{12}(x)\right\rangle$ \\
$p_{13}(x)=x^{8}+x^{7}+x^{6}+x^{5}+x^{2}+x+1 ; \alpha_{13}$ & $\mathbb{Z}_{2}[x] /\left\langle p_{13}(x)\right\rangle$ \\
$p_{14}(x)=x^{8}+x^{7}+x^{6}+x^{3}+x^{2}+x+1 ; \alpha_{14}$ & $\mathbb{Z}_{2}[x] /\left\langle p_{14}(x)\right\rangle$ \\
$p_{15}(x)=x^{8}+x^{7}+x^{6}+x^{5}+x^{4}+x^{2}+1 ; \alpha_{15}$ & $\mathbb{Z}_{2}[x] /\left\langle p_{15}(x)\right\rangle$ \\
$p_{16}(x)=x^{8}+x^{6}+x^{5}+x^{4}+1 ; \alpha_{16}$ & $\mathbb{Z}_{2}[x] /\left\langle p_{16}(x)\right\rangle$ \\
\hline
\end{tabular}

\section{Security Analysis}

In this section, we present some algebraic and statistical analyses of S-box followed [21]. Such analyses indicate the strength of all the proposed S-boxes and give an idea for their application in image encryption and other modes of secure communication.

3.1. Nonlinearity. Nonlinearity analysis of a function $f$ is the minimum hamming distance between the Boolean function $f: f:\{0,1\}^{n} \longrightarrow\{0,1\}$ and its all n-bit affine functions. In the truth table of Boolean function $f$, the nonlinearity of $f$ represents the degree of dissimilarity between $f$ and all affine function. If the function has high minimum hamming distance, it indicates it has high nonlinearity. It is an established fact that high nonlinearity provides resistance to any kind of linear approximation attacks $[22,23]$. The calculated upper bound of nonlinearity is $M=2^{m-1}-2^{((m / 2)-1)}$ so that, for $m=8$, the optimal value of nonlinearity is 120 . Table 18 shows the nonlinearity of $16 \mathrm{~S}$-boxes corresponding to all primitive irreducible polynomials. From this table, it can be seen that the value of nonlinearity has not been affected due to background irreducible polynomial.

3.2. Strict Avalanche Criteria. In [24], Webster and Tavares introduced the strict avalanche criteria (SAC) on the concepts of completeness and avalanche. If a single input bit changes, the output bits change with almost 0.5 probability. It helps to show that the resulting output vector is highly random, and no single pattern can be predictable by minor variation in the input vector [25]. By seeing the performance indexes of S-boxes, the proposed S-boxes successfully satisfy SAC. Table 19 depicts the value of SAC for all the proposed 16 S-boxes. It shows that the maximum value of SAC is 0.562500 for the first 9 S-boxes including $11^{\text {th }}, 14^{\text {th }}$, and $16^{\text {th }}$ S-boxes. Similarly, 


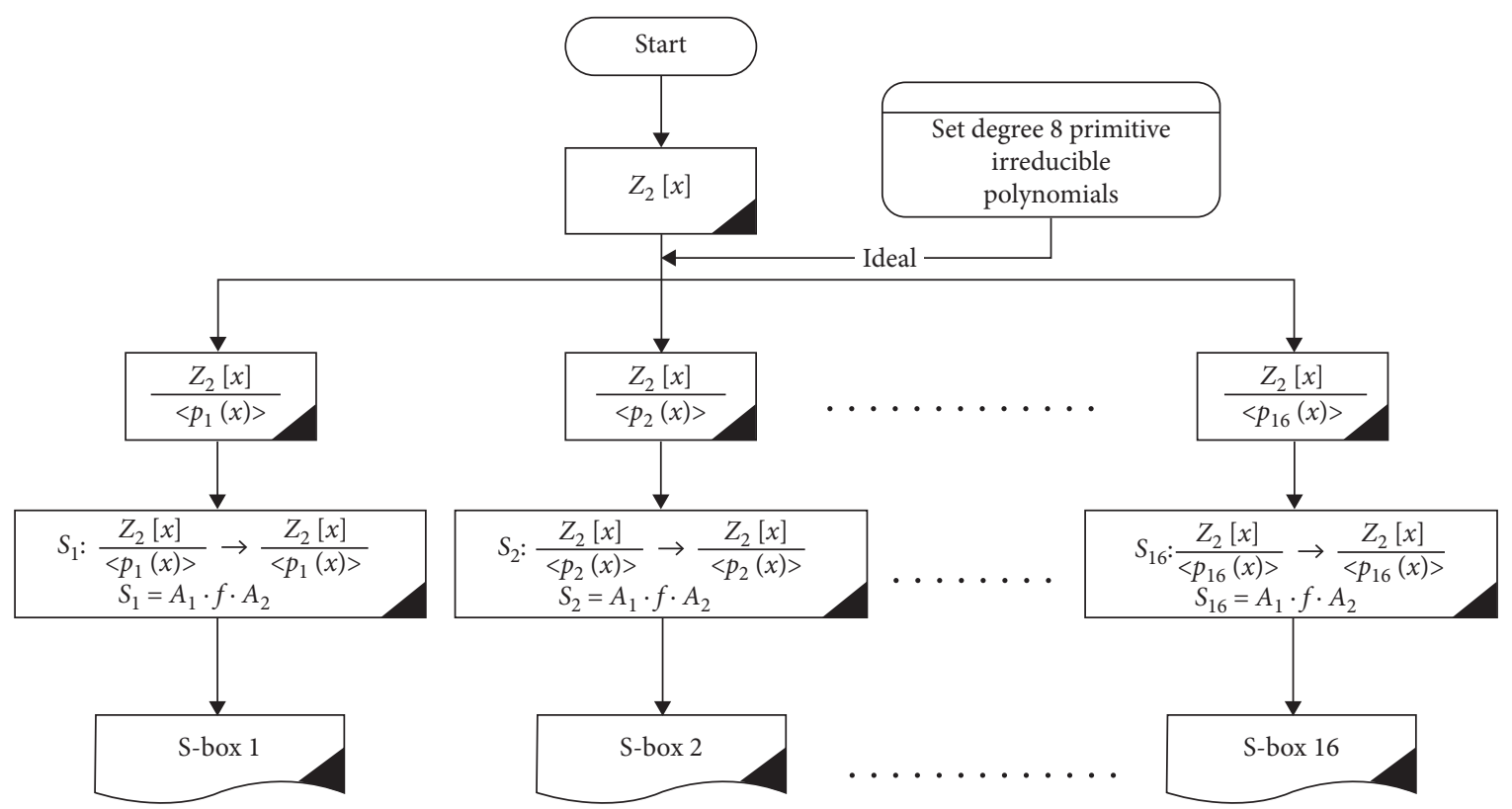

FIgURE 1: Flow chart for the construction of the proposed S-boxes and S-boxes corresponding to $P_{1}$ and $P_{16}$.

TABLE 2: S-box corresponding to P2.

\begin{tabular}{|c|c|c|c|c|c|c|c|c|c|c|c|c|c|c|c|}
\hline \multicolumn{16}{|c|}{ Proposed S-box 1} \\
\hline 176 & 141 & 139 & 249 & 75 & 179 & 4 & 69 & 48 & 62 & 243 & 197 & 49 & 105 & 167 & 250 \\
\hline 240 & 60 & 189 & 182 & 188 & 230 & 178 & 101 & 236 & 21 & 153 & 110 & 38 & 155 & 127 & 207 \\
\hline 91 & 213 & 29 & 55 & 88 & 78 & 244 & 215 & 81 & 221 & 158 & 219 & 74 & 201 & 210 & 255 \\
\hline 119 & 90 & 37 & 42 & 43 & 147 & 67 & 83 & 96 & 22 & 99 & 253 & 144 & 11 & 56 & 9 \\
\hline 63 & 223 & 205 & 80 & 140 & 71 & 121 & 125 & 120 & 54 & 168 & 187 & 82 & 202 & 10 & 70 \\
\hline 26 & 19 & 161 & 186 & 183 & 65 & 232 & 64 & 172 & 41 & 93 & 44 & 40 & 137 & 34 & 128 \\
\hline 164 & 241 & 163 & 25 & 87 & 214 & 76 & 196 & 1 & 31 & 45 & 8 & 198 & 97 & 102 & 246 \\
\hline 2 & 77 & 235 & 233 & 51 & 57 & 118 & 100 & 117 & 208 & 143 & 30 & 138 & 217 & 66 & 157 \\
\hline 211 & 245 & 15 & 134 & 180 & 126 & 114 & 142 & 89 & 148 & 254 & 218 & 123 & 85 & 154 & 169 \\
\hline 103 & 165 & 6 & 195 & 84 & 224 & 184 & 107 & 203 & 200 & 145 & 229 & 0 & 7 & 231 & 226 \\
\hline 58 & 3 & 53 & 212 & 191 & 159 & 35 & 242 & 5 & 220 & 216 & 247 & 192 & 204 & 136 & 228 \\
\hline 79 & 115 & 92 & 227 & 248 & 73 & 50 & 112 & 132 & 108 & 225 & 16 & 252 & 23 & 130 & 185 \\
\hline 33 & 133 & 237 & 61 & 173 & 171 & 27 & 86 & 113 & 181 & 175 & 116 & 251 & 177 & 170 & 234 \\
\hline 12 & 150 & 18 & 14 & 68 & 174 & 149 & 39 & 17 & 124 & 199 & 32 & 131 & 28 & 162 & 20 \\
\hline 160 & 111 & 135 & 13 & 104 & 129 & 239 & 98 & 222 & 109 & 146 & 72 & 152 & 206 & 190 & 238 \\
\hline 52 & 95 & 166 & 24 & 209 & 46 & 47 & 59 & 122 & 156 & 106 & 151 & 194 & 94 & 36 & 193 \\
\hline
\end{tabular}

TABLE 3: S-box corresponding to P2.

\begin{tabular}{|c|c|c|c|c|c|c|c|c|c|c|c|c|c|c|c|}
\hline \multicolumn{16}{|c|}{ Proposed S-box 2} \\
\hline 186 & 170 & 249 & 53 & 208 & 106 & 49 & 220 & 147 & 203 & 143 & 207 & 250 & 177 & 133 & 19 \\
\hline 240 & 84 & 152 & 38 & 18 & 21 & 58 & 59 & 67 & 219 & 15 & 209 & 70 & 150 & 41 & 121 \\
\hline 64 & 255 & 231 & 139 & 182 & 117 & 25 & 100 & 47 & 125 & 109 & 77 & 190 & 113 & 110 & 148 \\
\hline 118 & 197 & 198 & 146 & 39 & 138 & 108 & 161 & 75 & 13 & 103 & 200 & 73 & 91 & 105 & 204 \\
\hline 80 & 104 & 130 & 124 & 2 & 132 & 214 & 63 & 144 & 48 & 112 & 181 & 72 & 196 & 37 & 151 \\
\hline 3 & 135 & 10 & 129 & 221 & 184 & 26 & 111 & 40 & 222 & 11 & 127 & 223 & 31 & 29 & 87 \\
\hline 86 & 189 & 205 & 23 & 253 & 114 & 89 & 218 & 176 & 193 & 229 & 180 & 201 & 158 & 4 & 69 \\
\hline 22 & 188 & 228 & 85 & 241 & 236 & 140 & 239 & 16 & 164 & 57 & 79 & 8 & 56 & 24 & 102 \\
\hline 211 & 160 & 142 & 153 & 54 & 68 & 213 & 210 & 230 & 71 & 238 & 235 & 82 & 252 & 52 & 98 \\
\hline 33 & 7 & 65 & 42 & 5 & 1 & 32 & 162 & 212 & 192 & 175 & 90 & 17 & 245 & 43 & 119 \\
\hline 178 & 44 & 36 & 46 & 166 & 171 & 51 & 234 & 195 & 62 & 145 & 45 & 247 & 14 & 168 & 174 \\
\hline 217 & 122 & 159 & 0 & 101 & 237 & 60 & 27 & 163 & 202 & 243 & 99 & 116 & 120 & 131 & 6 \\
\hline 224 & 232 & 20 & 123 & 34 & 55 & 137 & 206 & 155 & 226 & 81 & 173 & 93 & 169 & 107 & 172 \\
\hline 167 & 199 & 191 & 94 & 136 & 96 & 183 & 149 & 233 & 246 & 179 & 156 & 9 & 83 & 50 & 95 \\
\hline 61 & 28 & 254 & 88 & 141 & 165 & 126 & 35 & 128 & 66 & 76 & 134 & 216 & 97 & 242 & 187 \\
\hline 244 & 30 & 74 & 215 & 12 & 115 & 185 & 194 & 78 & 154 & 227 & 251 & 225 & 248 & 92 & 157 \\
\hline
\end{tabular}


TABle 4: S-box corresponding to P3.

\begin{tabular}{|c|c|c|c|c|c|c|c|c|c|c|c|c|c|c|c|}
\hline \multicolumn{16}{|c|}{ Proposed S-box 3} \\
\hline 126 & 250 & 162 & 102 & 32 & 143 & 129 & 192 & 28 & 200 & 47 & 42 & 155 & 131 & 177 & 221 \\
\hline 240 & 142 & 29 & 49 & 138 & 120 & 94 & 46 & 20 & 64 & 5 & 99 & 116 & 27 & 50 & 167 \\
\hline 67 & 165 & 117 & 133 & 108 & 175 & 96 & 0 & 174 & 54 & 58 & 251 & 223 & 164 & 181 & 44 \\
\hline 154 & 88 & 238 & 69 & 89 & 194 & 201 & 193 & 22 & 75 & 61 & 137 & 123 & 72 & 86 & 169 \\
\hline 121 & 71 & 144 & 18 & 159 & 90 & 16 & 189 & 105 & 31 & 51 & 26 & 8 & 149 & 176 & 38 \\
\hline 65 & 110 & 48 & 183 & 210 & 196 & 217 & 59 & 163 & 237 & 118 & 93 & 13 & 241 & 140 & 208 \\
\hline 173 & 247 & 119 & 33 & 233 & 228 & 66 & 170 & 80 & 222 & 166 & 161 & 160 & 146 & 236 & 224 \\
\hline 179 & 63 & 115 & 81 & 98 & 231 & 150 & 97 & 243 & 229 & 178 & 215 & 17 & 6 & 14 & 245 \\
\hline 211 & 199 & 253 & 197 & 85 & 41 & 43 & 76 & 3 & 62 & 128 & 55 & 91 & 40 & 124 & 60 \\
\hline 73 & 134 & 77 & 21 & 1 & 107 & 218 & 180 & 204 & 112 & 15 & 230 & 246 & 104 & 151 & 130 \\
\hline 168 & 220 & 227 & 145 & 103 & 185 & 191 & 2 & 182 & 82 & 52 & 202 & 34 & 190 & 25 & 152 \\
\hline 139 & 114 & 187 & 186 & 141 & 30 & 122 & 113 & 23 & 92 & 111 & 101 & 12 & 239 & 127 & 213 \\
\hline 87 & 156 & 188 & 216 & 184 & 153 & 37 & 249 & 70 & 10 & 226 & 255 & 95 & 4 & 84 & 206 \\
\hline 148 & 214 & 172 & 225 & 254 & 45 & 135 & 24 & 35 & 11 & 53 & 39 & 195 & 9 & 252 & 248 \\
\hline 203 & 36 & 205 & 100 & 242 & 244 & 235 & 209 & 125 & 74 & 232 & 207 & 212 & 158 & 147 & 19 \\
\hline 109 & 56 & 219 & 157 & 171 & 7 & 106 & 57 & 234 & 83 & 136 & 79 & 132 & 68 & 198 & 78 \\
\hline
\end{tabular}

Table 5: S-box corresponding to P4.

\begin{tabular}{|c|c|c|c|c|c|c|c|c|c|c|c|c|c|c|c|}
\hline \multicolumn{16}{|c|}{ Proposed S-box 4} \\
\hline 49 & 103 & 220 & 208 & 58 & 70 & 173 & 238 & 242 & 160 & 154 & 169 & 158 & 110 & 139 & 94 \\
\hline 240 & 200 & 78 & 149 & 155 & 117 & 219 & 131 & 50 & 161 & 226 & 14 & 48 & 189 & 1 & 147 \\
\hline 115 & 120 & 218 & 34 & 51 & 7 & 104 & 182 & 167 & 150 & 192 & 64 & 178 & 95 & 57 & 91 \\
\hline 176 & 3 & 100 & 159 & 205 & 134 & 63 & 85 & 243 & 22 & 69 & 236 & 177 & 144 & 210 & 20 \\
\hline 44 & 79 & 206 & 89 & 96 & 74 & 66 & 146 & 197 & 15 & 0 & 108 & 13 & 215 & 25 & 181 \\
\hline 235 & 93 & 194 & 221 & 73 & 18 & 246 & 56 & 140 & 106 & 244 & 247 & 148 & 237 & 88 & 232 \\
\hline 191 & 251 & 234 & 99 & 9 & 33 & 59 & 252 & 45 & 179 & 222 & 125 & 132 & 109 & 229 & 136 \\
\hline 77 & 41 & 162 & 8 & 188 & 126 & 202 & 97 & 67 & 217 & 163 & 98 & 84 & 171 & 29 & 42 \\
\hline 211 & 38 & 213 & 119 & 46 & 168 & 5 & 116 & 204 & 129 & 75 & 31 & 152 & 239 & 166 & 172 \\
\hline 68 & 10 & 11 & 6 & 35 & 83 & 212 & 186 & 43 & 105 & 249 & 199 & 255 & 196 & 111 & 32 \\
\hline 87 & 184 & 156 & 24 & 223 & 190 & 195 & 153 & 92 & 230 & 112 & 209 & 39 & 52 & 80 & 12 \\
\hline 113 & 198 & 60 & 40 & 138 & 164 & 76 & 101 & 72 & 61 & 145 & 122 & 174 & 23 & 19 & 86 \\
\hline 170 & 183 & 214 & 245 & 135 & 231 & 36 & 54 & 224 & 81 & 143 & 124 & 30 & 228 & 4 & 114 \\
\hline 26 & 53 & 127 & 203 & 141 & 133 & 187 & 90 & 118 & 201 & 27 & 180 & 71 & 130 & 17 & 185 \\
\hline 82 & 175 & 137 & 165 & 28 & 216 & 227 & 37 & 55 & 47 & 2 & 157 & 248 & 233 & 123 & 254 \\
\hline 142 & 121 & 253 & 128 & 102 & 225 & 193 & 16 & 107 & 250 & 241 & 62 & 207 & 65 & 151 & 21 \\
\hline
\end{tabular}

TABLe 6: S-box corresponding to P5.

\begin{tabular}{|c|c|c|c|c|c|c|c|c|c|c|c|c|c|c|c|}
\hline \multicolumn{16}{|c|}{ Proposed S-box 5} \\
\hline 61 & 163 & 105 & 177 & 30 & 219 & 248 & 58 & 41 & 7 & 9 & 127 & 151 & 118 & 169 & 196 \\
\hline 240 & 34 & 202 & 51 & 77 & 191 & 126 & 233 & 215 & 39 & 254 & 197 & 20 & 48 & 93 & 192 \\
\hline 122 & 128 & 97 & 95 & 181 & 217 & 65 & 64 & 173 & 23 & 91 & 0 & 198 & 43 & 90 & 16 \\
\hline 175 & 87 & 70 & 162 & 168 & 92 & 2 & 49 & 100 & 245 & 193 & 249 & 205 & 107 & 55 & 139 \\
\hline 108 & 33 & 11 & 114 & 74 & 81 & 53 & 15 & 6 & 110 & 206 & 137 & 200 & 104 & 247 & 116 \\
\hline 19 & 182 & 14 & 46 & 60 & 115 & 158 & 167 & 130 & 113 & 17 & 204 & 4 & 218 & 141 & 176 \\
\hline 147 & 1 & 138 & 216 & 213 & 179 & 226 & 150 & 253 & 31 & 85 & 66 & 231 & 187 & 243 & 57 \\
\hline 71 & 236 & 244 & 225 & 45 & 38 & 165 & 40 & 172 & 201 & 188 & 119 & 224 & 242 & 29 & 232 \\
\hline 211 & 214 & 54 & 185 & 42 & 227 & 190 & 82 & 159 & 44 & 157 & 250 & 235 & 221 & 171 & 28 \\
\hline 155 & 35 & 52 & 124 & 120 & 84 & 78 & 136 & 59 & 123 & 36 & 88 & 251 & 83 & 134 & 21 \\
\hline 3 & 143 & 153 & 223 & 102 & 129 & 209 & 111 & 131 & 140 & 144 & 184 & 98 & 80 & 178 & 220 \\
\hline 12 & 148 & 186 & 99 & 149 & 210 & 239 & 94 & 234 & 37 & 152 & 230 & 63 & 241 & 67 & 189 \\
\hline 22 & 194 & 238 & 203 & 56 & 207 & 117 & 26 & 86 & 112 & 18 & 237 & 68 & 47 & 212 & 229 \\
\hline 166 & 146 & 199 & 228 & 121 & 101 & 180 & 174 & 76 & 72 & 32 & 160 & 69 & 208 & 10 & 125 \\
\hline 246 & 73 & 252 & 103 & 164 & 8 & 79 & 109 & 25 & 27 & 24 & 106 & 142 & 154 & 183 & 62 \\
\hline 96 & 255 & 132 & 156 & 75 & 145 & 50 & 135 & 13 & 133 & 222 & 161 & 170 & 5 & 195 & 89 \\
\hline
\end{tabular}


Table 7: S-box corresponding to P6.

\begin{tabular}{|c|c|c|c|c|c|c|c|c|c|c|c|c|c|c|c|}
\hline \multicolumn{16}{|c|}{ Proposed S-box 6} \\
\hline 162 & 120 & 205 & 183 & 16 & 20 & 137 & 191 & 89 & 95 & 128 & 239 & 159 & 10 & 219 & 94 \\
\hline 240 & 155 & 187 & 248 & 196 & 92 & 139 & 223 & 12 & 136 & 18 & 222 & 80 & 140 & 131 & 158 \\
\hline 100 & 53 & 236 & 50 & 192 & 72 & 118 & 167 & 132 & 42 & 3 & 51 & 90 & 138 & 15 & 28 \\
\hline 249 & 246 & 174 & 221 & 11 & 161 & 19 & 126 & 150 & 0 & 254 & 96 & 13 & 49 & 181 & 112 \\
\hline 81 & 163 & 108 & 26 & 135 & 216 & 234 & 99 & 85 & 14 & 78 & 62 & 177 & 104 & 179 & 207 \\
\hline 189 & 22 & 255 & 27 & 178 & 225 & 212 & 237 & 147 & 114 & 253 & 165 & 75 & 17 & 244 & 184 \\
\hline 199 & 166 & 87 & 208 & 71 & 242 & 195 & 175 & 101 & 247 & 79 & 243 & 149 & 44 & 217 & 201 \\
\hline 38 & 36 & 37 & 5 & 251 & 63 & 125 & 66 & 69 & 218 & 190 & 86 & 103 & 30 & 148 & 123 \\
\hline 211 & 198 & 33 & 48 & 232 & 197 & 105 & 9 & 93 & 31 & 107 & 145 & 194 & 185 & 41 & 227 \\
\hline 40 & 146 & 214 & 58 & 228 & 77 & 144 & 152 & 57 & 35 & 180 & 172 & 241 & 60 & 55 & 6 \\
\hline 8 & 61 & 24 & 229 & 169 & 1 & 168 & 171 & 154 & 204 & 52 & 233 & 21 & 213 & 119 & 245 \\
\hline 56 & 133 & 113 & 127 & 45 & 98 & 115 & 65 & 64 & 134 & 173 & 200 & 102 & 202 & 110 & 74 \\
\hline 182 & 157 & 141 & 76 & 91 & 68 & 34 & 224 & 59 & 203 & 206 & 170 & 215 & 153 & 73 & 39 \\
\hline 67 & 29 & 130 & 142 & 116 & 186 & 88 & 160 & 47 & 106 & 2 & 54 & 193 & 121 & 231 & 210 \\
\hline 7 & 252 & 117 & 250 & 176 & 4 & 84 & 43 & 124 & 235 & 209 & 151 & 122 & 230 & 226 & 129 \\
\hline 32 & 23 & 188 & 111 & 156 & 220 & 164 & 238 & 82 & 97 & 143 & 70 & 83 & 46 & 109 & 25 \\
\hline
\end{tabular}

TABle 8: S-box corresponding to P7.

\begin{tabular}{|c|c|c|c|c|c|c|c|c|c|c|c|c|c|c|c|}
\hline \multicolumn{16}{|c|}{ Proposed S-box 7} \\
\hline 86 & 101 & 177 & 89 & 199 & 188 & 236 & 165 & 198 & 145 & 112 & 232 & 92 & 164 & 76 & 137 \\
\hline 240 & 244 & 5 & 87 & 151 & 98 & 181 & 129 & 117 & 94 & 230 & 108 & 39 & 29 & 184 & 206 \\
\hline 103 & 123 & 56 & 180 & 35 & 142 & 246 & 168 & 48 & 36 & 64 & 222 & 187 & 111 & 196 & 15 \\
\hline 54 & 190 & 120 & 104 & 173 & 1 & 208 & 105 & 162 & 224 & 251 & 12 & 253 & 172 & 57 & 170 \\
\hline 114 & 3 & 153 & 159 & 185 & 204 & 19 & 245 & 128 & 50 & 97 & 140 & 227 & 127 & 214 & 44 \\
\hline 176 & 218 & 118 & 209 & 4 & 53 & 247 & 68 & 186 & 249 & 6 & 93 & 25 & 150 & 28 & 88 \\
\hline 0 & 254 & 115 & 147 & 85 & 154 & 221 & 99 & 69 & 45 & 70 & 136 & 130 & 32 & 8 & 134 \\
\hline 171 & 139 & 250 & 243 & 248 & 174 & 191 & 20 & 31 & 2 & 43 & 33 & 255 & 22 & 152 & 27 \\
\hline 211 & 71 & 61 & 109 & 95 & 47 & 9 & 226 & 135 & 67 & 143 & 124 & 160 & 125 & 228 & 18 \\
\hline 7 & 49 & 26 & 79 & 220 & 179 & 231 & 223 & 148 & 210 & 51 & 241 & 141 & 113 & 200 & 16 \\
\hline 192 & 197 & 239 & 146 & 24 & 183 & 252 & 82 & 167 & 126 & 225 & 242 & 58 & 219 & 52 & 107 \\
\hline 233 & 55 & 149 & 235 & 77 & 178 & 157 & 201 & 65 & 40 & 216 & 74 & 207 & 133 & 131 & 78 \\
\hline 83 & 122 & 73 & 102 & 66 & 213 & 138 & 80 & 11 & 182 & 34 & 46 & 195 & 10 & 193 & 17 \\
\hline 237 & 75 & 119 & 110 & 238 & 156 & 21 & 72 & 203 & 175 & 234 & 41 & 189 & 158 & 116 & 215 \\
\hline 205 & 13 & 91 & 202 & 106 & 38 & 229 & 30 & 81 & 23 & 37 & 63 & 144 & 96 & 59 & 42 \\
\hline 62 & 100 & 84 & 166 & 14 & 60 & 217 & 161 & 169 & 132 & 163 & 90 & 212 & 194 & 155 & 121 \\
\hline
\end{tabular}

TABle 9: S-box corresponding to P8.

\begin{tabular}{|c|c|c|c|c|c|c|c|c|c|c|c|c|c|c|c|}
\hline \multicolumn{16}{|c|}{ Proposed S-box 8} \\
\hline 41 & 6 & 47 & 28 & 109 & 248 & 100 & 139 & 227 & 141 & 111 & 60 & 7 & 125 & 178 & 31 \\
\hline 240 & 224 & 53 & 123 & 91 & 63 & 67 & 237 & 71 & 217 & 11 & 205 & 252 & 133 & 61 & 22 \\
\hline 97 & 172 & 182 & 233 & 124 & 51 & 196 & 236 & 255 & 170 & 128 & 3 & 186 & 179 & 83 & 119 \\
\hline 29 & 206 & 234 & 210 & 218 & 1 & 151 & 220 & 245 & 68 & 13 & 213 & 54 & 214 & 30 & 15 \\
\hline 23 & 98 & 95 & 82 & 189 & 212 & 48 & 120 & 187 & 219 & 239 & 222 & 202 & 192 & 154 & 246 \\
\hline 143 & 24 & 131 & 181 & 102 & 27 & 204 & 184 & 129 & 137 & 114 & 74 & 122 & 57 & 188 & 199 \\
\hline 94 & 75 & 20 & 79 & 76 & 158 & 92 & 130 & 39 & 19 & 96 & 134 & 103 & 108 & 33 & 229 \\
\hline 89 & 21 & 175 & 50 & 166 & 195 & 87 & 203 & 164 & 46 & 174 & 121 & 25 & 34 & 241 & 16 \\
\hline 211 & 238 & 104 & 112 & 235 & 14 & 116 & 251 & 191 & 86 & 253 & 230 & 225 & 52 & 155 & 38 \\
\hline 58 & 177 & 173 & 40 & 45 & 148 & 62 & 42 & 201 & 149 & 190 & 250 & 216 & 157 & 59 & 244 \\
\hline 169 & 153 & 150 & 117 & 142 & 101 & 145 & 9 & 207 & 208 & 247 & 160 & 140 & 8 & 64 & 180 \\
\hline 194 & 88 & 37 & 162 & 115 & 35 & 156 & 5 & 90 & 36 & 84 & 106 & 152 & 232 & 159 & 110 \\
\hline 144 & 73 & 80 & 126 & 200 & 165 & 113 & 56 & 161 & 49 & 127 & 12 & 168 & 0 & 66 & 226 \\
\hline 147 & 32 & 132 & 146 & 243 & 4 & 43 & 138 & 55 & 163 & 167 & 135 & 223 & 81 & 93 & 209 \\
\hline 69 & 99 & 107 & 44 & 198 & 105 & 228 & 10 & 65 & 77 & 215 & 197 & 193 & 176 & 18 & 17 \\
\hline 249 & 78 & 242 & 85 & 70 & 72 & 171 & 183 & 26 & 221 & 254 & 231 & 118 & 136 & 185 & 2 \\
\hline
\end{tabular}


Table 10: S-box corresponding to P9.

\begin{tabular}{|c|c|c|c|c|c|c|c|c|c|c|c|c|c|c|c|}
\hline \multicolumn{16}{|c|}{ Proposed S-box 9} \\
\hline 164 & 17 & 78 & 216 & 114 & 139 & 98 & 51 & 31 & 89 & 58 & 243 & 221 & 249 & 159 & 18 \\
\hline 240 & 64 & 151 & 225 & 120 & 210 & 180 & 135 & 57 & 115 & 204 & 72 & 62 & 155 & 227 & 195 \\
\hline 109 & 95 & 127 & 141 & 76 & 229 & 178 & 246 & 12 & 147 & 47 & 235 & 166 & 217 & 122 & 212 \\
\hline 205 & 26 & 27 & 138 & 148 & 70 & 171 & 130 & 140 & 215 & 203 & 156 & 146 & 11 & 106 & 233 \\
\hline 242 & 162 & 6 & 206 & 142 & 16 & 119 & 218 & 253 & 169 & 104 & 213 & 102 & 41 & 245 & 231 \\
\hline 197 & 250 & 65 & 86 & 90 & 152 & 237 & 241 & 167 & 254 & 4 & 14 & 208 & 182 & 71 & 101 \\
\hline 232 & 68 & 111 & 10 & 134 & 55 & 209 & 103 & 189 & 61 & 74 & 116 & 20 & 40 & 118 & 186 \\
\hline 42 & 192 & 131 & 226 & 43 & 113 & 63 & 157 & 38 & 69 & 8 & 60 & 149 & 44 & 255 & 53 \\
\hline 211 & 82 & 224 & 110 & 32 & 94 & 49 & 236 & 80 & 160 & 196 & 222 & 185 & 108 & 188 & 154 \\
\hline 176 & 123 & 238 & 117 & 30 & 247 & 88 & 150 & 181 & 144 & 132 & 35 & 37 & 198 & 96 & 97 \\
\hline 136 & 92 & 23 & 75 & 121 & 81 & 112 & 83 & 125 & 239 & 87 & 2 & 143 & 19 & 105 & 201 \\
\hline 137 & 214 & 15 & 56 & 172 & 179 & 133 & 252 & 36 & 220 & 85 & 184 & 50 & 234 & 39 & 194 \\
\hline 73 & 99 & 173 & 79 & 124 & 46 & 25 & 100 & 168 & 3 & 33 & 228 & 191 & 145 & 165 & 161 \\
\hline 199 & 77 & 126 & 28 & 183 & 59 & 170 & 22 & 54 & 202 & 223 & 66 & 163 & 219 & 187 & 174 \\
\hline 177 & 244 & 175 & 5 & 128 & 67 & 230 & 7 & 93 & 45 & 29 & 21 & 158 & 13 & 129 & 9 \\
\hline 0 & 48 & 200 & 107 & 34 & 24 & 251 & 248 & 1 & 190 & 84 & 52 & 193 & 153 & 91 & 207 \\
\hline
\end{tabular}

Table 11: S-box corresponding to P10.

\begin{tabular}{|c|c|c|c|c|c|c|c|c|c|c|c|c|c|c|c|}
\hline \multicolumn{16}{|c|}{ Proposed S-box 10} \\
\hline 109 & 26 & 163 & 213 & 77 & 207 & 155 & 87 & 34 & 96 & 136 & 40 & 177 & 25 & 128 & 11 \\
\hline 240 & 147 & 166 & 252 & 115 & 158 & 185 & 123 & 146 & 68 & 239 & 149 & 160 & 180 & 6 & 111 \\
\hline 22 & 198 & 208 & 243 & 103 & 97 & 24 & 187 & 179 & 228 & 132 & 110 & 188 & 10 & 151 & 130 \\
\hline 62 & 119 & 131 & 60 & 219 & 148 & 245 & 9 & 101 & 81 & 205 & 3 & 222 & 98 & 203 & 19 \\
\hline 154 & 178 & 66 & 117 & 246 & 108 & 226 & 135 & 202 & 224 & 59 & 236 & 192 & 156 & 141 & 112 \\
\hline 217 & 28 & 39 & 167 & 172 & 104 & 44 & 230 & 54 & 253 & 82 & 168 & 23 & 4 & 150 & 58 \\
\hline 107 & 64 & 43 & 48 & 20 & 118 & 181 & 102 & 173 & 254 & 200 & 76 & 237 & 209 & 143 & 204 \\
\hline 63 & 193 & 221 & 214 & 14 & 88 & 16 & 126 & 223 & 90 & 157 & 85 & 46 & 169 & 196 & 191 \\
\hline 211 & 42 & 1 & 199 & 176 & 100 & 216 & 152 & 183 & 89 & 37 & 99 & 75 & 233 & 72 & 122 \\
\hline 184 & 52 & 79 & 206 & 235 & 194 & 225 & 71 & 15 & 162 & 50 & 210 & 242 & 127 & 153 & 234 \\
\hline 57 & 70 & 165 & 248 & 164 & 5 & 67 & 55 & 2 & 129 & 124 & 189 & 21 & 171 & 241 & 86 \\
\hline 251 & 159 & 125 & 134 & 32 & 93 & 116 & 12 & 255 & 106 & 175 & 73 & 170 & 35 & 139 & 84 \\
\hline 41 & 120 & 18 & 27 & 78 & 17 & 182 & 174 & 53 & 215 & 83 & 247 & 94 & 227 & 31 & 137 \\
\hline 29 & 61 & 113 & 145 & 212 & 238 & 138 & 105 & 30 & 47 & 121 & 92 & 74 & 7 & 80 & 142 \\
\hline 232 & 95 & 197 & 220 & 195 & 231 & 49 & 161 & 36 & 8 & 69 & 91 & 65 & 13 & 244 & 218 \\
\hline 51 & 0 & 250 & 144 & 229 & 114 & 190 & 45 & 249 & 133 & 140 & 38 & 33 & 186 & 201 & 56 \\
\hline
\end{tabular}

Table 12: S-box corresponding to P11.

\begin{tabular}{|c|c|c|c|c|c|c|c|c|c|c|c|c|c|c|c|}
\hline \multicolumn{16}{|c|}{ Proposed S-box 11} \\
\hline 128 & 191 & 132 & 167 & 111 & 120 & 159 & 218 & 25 & 68 & 173 & 217 & 32 & 39 & 99 & 239 \\
\hline 240 & 94 & 157 & 95 & 75 & 112 & 190 & 213 & 152 & 202 & 40 & 101 & 2 & 207 & 140 & 64 \\
\hline 19 & 238 & 35 & 151 & 154 & 197 & 199 & 60 & 61 & 187 & 44 & 201 & 72 & 37 & 126 & 118 \\
\hline 24 & 80 & 124 & 141 & 16 & 41 & 193 & 160 & 7 & 107 & 163 & 129 & 248 & 66 & 189 & 221 \\
\hline 10 & 109 & 76 & 150 & 110 & 255 & 171 & 70 & 17 & 18 & 43 & 174 & 153 & 13 & 113 & 206 \\
\hline 244 & 219 & 3 & 203 & 12 & 134 & 48 & 245 & 164 & 130 & 230 & 4 & 144 & 53 & 182 & 78 \\
\hline 155 & 227 & 253 & 158 & 26 & 86 & 186 & 62 & 79 & 21 & 175 & 162 & 222 & 215 & 247 & 208 \\
\hline 98 & 137 & 210 & 63 & 57 & 67 & 138 & 96 & 139 & 242 & 254 & 38 & 92 & 188 & 97 & 52 \\
\hline 211 & 106 & 6 & 9 & 22 & 85 & 212 & 198 & 234 & 225 & 42 & 223 & 119 & 136 & 90 & 33 \\
\hline 46 & 121 & 249 & 184 & 34 & 142 & 251 & 20 & 214 & 216 & 50 & 209 & 135 & 87 & 74 & 176 \\
\hline 161 & 5 & 77 & 36 & 229 & 83 & 149 & 73 & 168 & 181 & 196 & 115 & 194 & 11 & 56 & 8 \\
\hline 82 & 231 & 51 & 1 & 148 & 65 & 195 & 172 & 23 & 177 & 228 & 0 & 131 & 252 & 88 & 170 \\
\hline 220 & 27 & 69 & 71 & 123 & 232 & 49 & 226 & 236 & 58 & 104 & 143 & 178 & 166 & 117 & 205 \\
\hline 54 & 169 & 108 & 91 & 204 & 127 & 116 & 45 & 165 & 89 & 30 & 55 & 31 & 84 & 246 & 15 \\
\hline 156 & 122 & 224 & 192 & 103 & 145 & 243 & 114 & 185 & 102 & 200 & 180 & 250 & 81 & 233 & 47 \\
\hline 59 & 125 & 133 & 100 & 147 & 14 & 183 & 146 & 235 & 237 & 29 & 28 & 105 & 93 & 179 & 241 \\
\hline
\end{tabular}


TABLe 13: S-box corresponding to P12.

\begin{tabular}{|c|c|c|c|c|c|c|c|c|c|c|c|c|c|c|c|}
\hline \multicolumn{16}{|c|}{ Proposed S-box 12} \\
\hline 140 & 249 & 110 & 3 & 253 & 6 & 19 & 160 & 102 & 247 & 235 & 219 & 17 & 166 & 184 & 34 \\
\hline 240 & 198 & 221 & 159 & 212 & 199 & 188 & 148 & 27 & 0 & 52 & 73 & 176 & 15 & 4 & 93 \\
\hline 1 & 28 & 32 & 214 & 195 & 238 & 216 & 24 & 229 & 92 & 241 & 36 & 209 & 207 & 12 & 9 \\
\hline 25 & 245 & 139 & 97 & 109 & 41 & 225 & 37 & 105 & 106 & 71 & 100 & 206 & 165 & 129 & 59 \\
\hline 171 & 70 & 208 & 126 & 111 & 242 & 181 & 119 & 215 & 22 & 183 & 233 & 14 & 222 & 204 & 150 \\
\hline 226 & 144 & 82 & 121 & 40 & 87 & 67 & 228 & 116 & 234 & 81 & 13 & 180 & 5 & 96 & 237 \\
\hline 42 & 51 & 95 & 16 & 99 & 79 & 21 & 69 & 31 & 30 & 125 & 55 & 89 & 143 & 123 & 178 \\
\hline 122 & 158 & 80 & 66 & 10 & 133 & 46 & 252 & 33 & 112 & 210 & 157 & 131 & 203 & 127 & 43 \\
\hline 211 & 54 & 132 & 217 & 58 & 155 & 152 & 192 & 53 & 128 & 84 & 44 & 88 & 236 & 194 & 168 \\
\hline 170 & 239 & 231 & 218 & 232 & 205 & 103 & 151 & 2 & 173 & 26 & 145 & 20 & 251 & 136 & 117 \\
\hline 146 & 49 & 83 & 35 & 202 & 90 & 243 & 164 & 78 & 108 & 201 & 167 & 175 & 191 & 68 & 163 \\
\hline 65 & 85 & 185 & 255 & 104 & 18 & 227 & 177 & 75 & 39 & 161 & 182 & 187 & 57 & 98 & 186 \\
\hline 64 & 246 & 179 & 196 & 174 & 38 & 118 & 135 & 101 & 193 & 77 & 223 & 94 & 248 & 72 & 250 \\
\hline 107 & 62 & 189 & 153 & 8 & 48 & 7 & 56 & 169 & 60 & 23 & 11 & 149 & 141 & 29 & 156 \\
\hline 147 & 120 & 154 & 254 & 134 & 130 & 244 & 200 & 172 & 213 & 197 & 162 & 190 & 113 & 45 & 86 \\
\hline 114 & 230 & 47 & 138 & 61 & 115 & 220 & 50 & 137 & 224 & 76 & 63 & 142 & 124 & 91 & 74 \\
\hline
\end{tabular}

Table 14: S-box corresponding to P13.

\begin{tabular}{|c|c|c|c|c|c|c|c|c|c|c|c|c|c|c|c|}
\hline \multicolumn{16}{|c|}{ Proposed S-box 13} \\
\hline 24 & 177 & 151 & 25 & 7 & 71 & 226 & 153 & 127 & 137 & 209 & 73 & 254 & 213 & 96 & 237 \\
\hline 240 & 234 & 102 & 131 & 201 & 142 & 118 & 64 & 16 & 249 & 176 & 6 & 5 & 18 & 222 & 95 \\
\hline 52 & 36 & 82 & 160 & 224 & 225 & 144 & 74 & 67 & 129 & 1 & 13 & 105 & 143 & 186 & 248 \\
\hline 181 & 113 & 231 & 108 & 58 & 216 & 43 & 114 & 156 & 37 & 168 & 9 & 170 & 60 & 3 & 246 \\
\hline 40 & 122 & 217 & 247 & 223 & 85 & 163 & 4 & 65 & 152 & 31 & 189 & 70 & 12 & 173 & 210 \\
\hline 207 & 233 & 197 & 107 & 83 & 115 & 164 & 99 & 66 & 56 & 23 & 111 & 120 & 61 & 251 & 46 \\
\hline 205 & 154 & 255 & 167 & 198 & 81 & 87 & 109 & 20 & 135 & 59 & 68 & 100 & 39 & 220 & 172 \\
\hline 145 & 11 & 132 & 185 & 106 & 53 & 162 & 112 & 253 & 54 & 180 & 33 & 116 & 175 & 104 & 134 \\
\hline 211 & 123 & 204 & 26 & 190 & 90 & 8 & 92 & 15 & 110 & 119 & 29 & 188 & 230 & 219 & 199 \\
\hline 76 & 79 & 174 & 130 & 147 & 166 & 63 & 215 & 241 & 41 & 244 & 141 & 124 & 0 & 35 & 98 \\
\hline 218 & 245 & 242 & 72 & 21 & 239 & 47 & 94 & 208 & 238 & 10 & 165 & 171 & 91 & 14 & 192 \\
\hline 77 & 243 & 148 & 158 & 80 & 203 & 45 & 42 & 194 & 146 & 159 & 235 & 214 & 161 & 32 & 169 \\
\hline 93 & 101 & 140 & 30 & 97 & 50 & 84 & 126 & 229 & 196 & 200 & 48 & 55 & 139 & 57 & 44 \\
\hline 69 & 128 & 49 & 155 & 19 & 62 & 232 & 252 & 236 & 136 & 250 & 27 & 157 & 195 & 125 & 206 \\
\hline 34 & 179 & 78 & 38 & 193 & 227 & 28 & 184 & 133 & 88 & 187 & 178 & 75 & 212 & 138 & 117 \\
\hline 191 & 202 & 149 & 22 & 228 & 89 & 150 & 121 & 221 & 17 & 182 & 86 & 51 & 183 & 103 & 2 \\
\hline
\end{tabular}

TABle 15: S-box corresponding to P14.

\begin{tabular}{|c|c|c|c|c|c|c|c|c|c|c|c|c|c|c|c|}
\hline \multicolumn{16}{|c|}{ Proposed S-box 14} \\
\hline 93 & 13 & 118 & 253 & 48 & 210 & 140 & 163 & 100 & 34 & 18 & 128 & 11 & 90 & 53 & 114 \\
\hline 240 & 227 & 242 & 106 & 112 & 138 & 147 & 92 & 250 & 206 & 141 & 56 & 152 & 57 & 94 & 212 \\
\hline 50 & 149 & 235 & 21 & 233 & 254 & 102 & 131 & 156 & 142 & 173 & 194 & 31 & 45 & 244 & 195 \\
\hline 237 & 43 & 183 & 168 & 121 & 95 & 9 & 71 & 37 & 120 & 204 & 62 & 119 & 174 & 122 & 186 \\
\hline 123 & 29 & 105 & 136 & 191 & 196 & 91 & 40 & 229 & 86 & 4 & 41 & 125 & 22 & 224 & 162 \\
\hline 155 & 30 & 223 & 161 & 39 & 169 & 14 & 63 & 52 & 133 & 199 & 36 & 241 & 124 & 59 & 65 \\
\hline 190 & 127 & 219 & 88 & 214 & 115 & 126 & 96 & 38 & 239 & 55 & 7 & 197 & 73 & 2 & 42 \\
\hline 23 & 144 & 47 & 46 & 68 & 72 & 6 & 185 & 109 & 176 & 1 & 98 & 201 & 234 & 222 & 203 \\
\hline 211 & 175 & 157 & 113 & 110 & 217 & 85 & 3 & 249 & 255 & 116 & 104 & 25 & 82 & 179 & 97 \\
\hline 158 & 135 & 44 & 78 & 117 & 164 & 160 & 221 & 66 & 236 & 145 & 103 & 60 & 230 & 232 & 134 \\
\hline 182 & 67 & 177 & 188 & 15 & 8 & 107 & 79 & 216 & 12 & 246 & 51 & 213 & 180 & 58 & 17 \\
\hline 189 & 252 & 215 & 245 & 69 & 218 & 192 & 10 & 225 & 74 & 54 & 83 & 84 & 61 & 166 & 193 \\
\hline 16 & 139 & 81 & 132 & 19 & 101 & 76 & 208 & 231 & 151 & 181 & 49 & 187 & 99 & 202 & 228 \\
\hline 205 & 220 & 154 & 198 & 111 & 167 & 20 & 26 & 200 & 64 & 165 & 247 & 248 & 209 & 207 & 148 \\
\hline 226 & 0 & 28 & 184 & 33 & 159 & 27 & 153 & 24 & 172 & 171 & 75 & 238 & 243 & 77 & 87 \\
\hline 35 & 170 & 32 & 137 & 143 & 251 & 129 & 178 & 146 & 150 & 80 & 89 & 108 & 130 & 5 & 70 \\
\hline
\end{tabular}


TABLE 16: S-box corresponding to P15.

\begin{tabular}{|c|c|c|c|c|c|c|c|c|c|c|c|c|c|c|c|}
\hline \multicolumn{16}{|c|}{ Proposed S-box 15} \\
\hline 12 & 220 & 182 & 7 & 142 & 239 & 96 & 251 & 36 & 246 & 21 & 162 & 223 & 103 & 35 & 40 \\
\hline 240 & 132 & 32 & 219 & 116 & 160 & 243 & 28 & 128 & 168 & 159 & 206 & 193 & 95 & 50 & 154 \\
\hline 38 & 234 & 47 & 66 & 126 & 192 & 54 & 169 & 65 & 175 & 254 & 89 & 45 & 123 & 233 & 39 \\
\hline 17 & 194 & 111 & 202 & 242 & 226 & 178 & 210 & 185 & 64 & 77 & 218 & 108 & 127 & 147 & 222 \\
\hline 191 & 214 & 237 & 110 & 184 & 155 & 30 & 20 & 86 & 212 & 156 & 1 & 255 & 119 & 167 & 141 \\
\hline 105 & 252 & 176 & 63 & 205 & 139 & 151 & 53 & 33 & 189 & 170 & 213 & 183 & 224 & 204 & 75 \\
\hline 69 & 100 & 230 & 196 & 188 & 31 & 43 & 104 & 83 & 91 & 145 & 60 & 113 & 117 & 152 & 94 \\
\hline 157 & 129 & 102 & 235 & 135 & 49 & 15 & 181 & 163 & 195 & 58 & 249 & 134 & 171 & 107 & 37 \\
\hline 211 & 34 & 24 & 238 & 67 & 22 & 236 & 88 & 253 & 109 & 207 & 221 & 164 & 203 & 138 & 19 \\
\hline 48 & 229 & 122 & 71 & 76 & 93 & 120 & 143 & 81 & 106 & 26 & 137 & 215 & 200 & 187 & 217 \\
\hline 118 & 72 & 244 & 245 & 150 & 209 & 121 & 144 & 199 & 14 & 186 & 27 & 70 & 115 & 84 & 55 \\
\hline 4 & 11 & 73 & 80 & 179 & 79 & 146 & 18 & 173 & 6 & 172 & 8 & 56 & 136 & 78 & 61 \\
\hline 42 & 52 & 85 & 166 & 133 & 41 & 228 & 25 & 99 & 90 & 158 & 125 & 57 & 29 & 2 & 248 \\
\hline 10 & 231 & 92 & 5 & 201 & 180 & 68 & 177 & 174 & 23 & 161 & 208 & 98 & 62 & 232 & 140 \\
\hline 51 & 165 & 216 & 0 & 241 & 101 & 9 & 97 & 131 & 148 & 198 & 247 & 130 & 114 & 250 & 112 \\
\hline 44 & 46 & 225 & 13 & 153 & 3 & 16 & 82 & 190 & 124 & 59 & 197 & 149 & 227 & 74 & 87 \\
\hline
\end{tabular}

Table 17: S-box corresponding to P15.

\begin{tabular}{|c|c|c|c|c|c|c|c|c|c|c|c|c|c|c|c|}
\hline \multicolumn{16}{|c|}{ Proposed S-box 16} \\
\hline 6 & 136 & 68 & 218 & 166 & 118 & 48 & 114 & 2 & 173 & 30 & 39 & 204 & 81 & 144 & 134 \\
\hline 240 & 46 & 115 & 83 & 91 & 202 & 1 & 203 & 10 & 63 & 145 & 4 & 89 & 112 & 213 & 17 \\
\hline 47 & 8 & 176 & 149 & 117 & 181 & 38 & 133 & 56 & 87 & 162 & 20 & 199 & 78 & 60 & 157 \\
\hline 254 & 14 & 192 & 29 & 200 & 178 & 140 & 69 & 24 & 121 & 160 & 148 & 158 & 252 & 67 & 228 \\
\hline 111 & 103 & 102 & 194 & 33 & 122 & 44 & 205 & 61 & 100 & 229 & 215 & 80 & 187 & 217 & 189 \\
\hline 28 & 124 & 147 & 92 & 31 & 76 & 45 & 171 & 137 & 239 & 95 & 154 & 42 & 64 & 51 & 55 \\
\hline 119 & 236 & 77 & 242 & 0 & 12 & 75 & 216 & 84 & 151 & 220 & 49 & 101 & 150 & 129 & 53 \\
\hline 155 & 198 & 174 & 146 & 90 & 233 & 88 & 225 & 54 & 179 & 5 & 74 & 246 & 138 & 110 & 230 \\
\hline 211 & 153 & 208 & 214 & 40 & 184 & 13 & 255 & 71 & 163 & 62 & 130 & 175 & 232 & 231 & 128 \\
\hline 156 & 141 & 34 & 152 & 15 & 212 & 165 & 41 & 106 & 97 & 167 & 72 & 177 & 169 & 234 & 107 \\
\hline 170 & 120 & 247 & 104 & 245 & 195 & 7 & 172 & 19 & 65 & 85 & 36 & 70 & 108 & 209 & 222 \\
\hline 197 & 132 & 244 & 57 & 201 & 182 & 96 & 52 & 125 & 210 & 183 & 127 & 241 & 126 & 235 & 26 \\
\hline 58 & 227 & 143 & 11 & 238 & 86 & 135 & 191 & 66 & 168 & 237 & 3 & 59 & 123 & 32 & 27 \\
\hline 105 & 207 & 221 & 22 & 248 & 251 & 243 & 50 & 23 & 16 & 186 & 98 & 82 & 250 & 180 & 164 \\
\hline 193 & 219 & 93 & 79 & 35 & 196 & 249 & 188 & 139 & 253 & 161 & 99 & 37 & 223 & 224 & 226 \\
\hline 131 & 21 & 109 & 25 & 73 & 116 & 43 & 206 & 94 & 113 & 190 & 142 & 185 & 159 & 9 & 18 \\
\hline
\end{tabular}

TABLE 18: Nonlinearity of the proposed S-box.

\begin{tabular}{lcccccccc}
\hline S-boxes & $f_{1}$ & $f_{2}$ & $f_{3}$ & $f_{4}$ & $f_{5}$ & $f_{6}$ & $f_{7}$ & $f_{8}$ \\
\hline S-box 1 & 112 & 112 & 112 & 112 & 112 & 112 & 112 & 112 \\
S-box 2 & 112 & 112 & 112 & 112 & 112 & 112 & 112 & 112 \\
S-box 3 & 112 & 112 & 112 & 112 & 112 & 112 & 112 & 112 \\
S-box 4 & 112 & 112 & 112 & 112 & 112 & 112 & 112 & 112 \\
S-box 5 & 112 & 112 & 112 & 112 & 112 & 112 & 112 & 112 \\
S-box 6 & 112 & 112 & 112 & 112 & 112 & 112 & 112 & 112 \\
S-box 7 & 112 & 112 & 112 & 112 & 112 & 112 & 112 & 112 \\
S-box 8 & 112 & 112 & 112 & 112 & 112 & 112 & 112 & 112 \\
S-box 9 & 112 & 112 & 112 & 112 & 112 & 112 & 112 & 112 \\
S-box 10 & 112 & 112 & 112 & 112 & 112 & 112 & 112 & 112 \\
S-box 11 & 112 & 112 & 112 & 112 & 112 & 112 & 112 & 112 \\
S-box 12 & 112 & 112 & 112 & 112 & 112 & 112 & 112 & 112 \\
S-box 13 & 112 & 112 & 112 & 112 & 112 & 112 & 112 & 112 \\
S-box 14 & 112 & 112 & 112 & 112 & 112 & 112 & 112 & 112 \\
S-box 15 & 112 & 112 & 112 & 112 & 112 & 112 & 112 & 112 \\
S-box 16 & 112 & 112 & 112 & 112 & 112 & 112 & 112 & 112 \\
\hline
\end{tabular}


TABle 19: Strict avalanche criterion.

\begin{tabular}{lcccc}
\hline S-box & Max & Min & Average & Square deviation \\
\hline S-box 1 & 0.562500 & 0.437500 & 0.496094 & 0.0172495 \\
S-box 2 & 0.546875 & 0.453125 & 0.495117 & 0.0128725 \\
S-box 3 & 0.562500 & 0.453125 & 0.494141 & 0.0152856 \\
S-box 4 & 0.562500 & 0.453125 & 0.50708 & 0.0118748 \\
S-box 5 & 0.546875 & 0.453125 & 0.503174 & 0.0153901 \\
S-box 6 & 0.562500 & 0.453125 & 0.501709 & 0.016637 \\
S-box 7 & 0.562500 & 0.453125 & 0.502441 & 0.0170951 \\
S-box 8 & 0.562500 & 0.453125 & 0.503906 & 0.0165152 \\
S-box 9 & 0.562500 & 0.453125 & 0.485596 & 0.0153978 \\
S-box 10 & 0.546875 & 0.453125 & 0.509766 & 0.0123912 \\
S-box 11 & 0.562500 & 0.4575 & 0.50125 & 0.0191487 \\
S-box 12 & 0.5625 & 0.4375 & 0.500977 & 0.016475 \\
S-box 13 & 0.546875 & 0.453125 & 0.508301 & 0.0127235 \\
S-box 14 & 0.5625 & 0.437500 & 0.498779 & 0.0158654 \\
S-box 15 & 0.546875 & 0.437500 & 0.496582 & 0.0143727 \\
S-box 16 & 0.5625 & & 0.0143171 \\
\hline
\end{tabular}

TABle 20: Bit independent criterion.

\begin{tabular}{|c|c|c|c|c|c|c|}
\hline \multicolumn{4}{|c|}{ BIC-SAC } & \multicolumn{3}{|c|}{$\mathrm{BIC}$} \\
\hline S-box & Min & Average & Square deviation & Max & Average & Square deviation \\
\hline S-box 1 & 0.47461 & 0.50119 & 0.01132 & 112 & 112 & 0 \\
\hline S-box 2 & 0.49219 & 0.50600 & 0.00845 & 112 & 112 & 0 \\
\hline S-box 3 & 0.48047 & 0.50202 & 0.01015 & 112 & 112 & 0 \\
\hline S-box 4 & 0.47852 & 0.50656 & 0.01201 & 112 & 112 & 0 \\
\hline S-box 5 & 0.48438 & 0.50105 & 0.00924 & 112 & 112 & 0 \\
\hline S-box 6 & 0.47656 & 0.49819 & 0.00784 & 112 & 112 & 0 \\
\hline S-box 7 & 0.48633 & 0.50593 & 0.00925 & 112 & 112 & 0 \\
\hline S-box 8 & 0.48828 & 0.50251 & 0.00835 & 112 & 112 & 0 \\
\hline S-box 9 & 0.48828 & 0.50258 & 0.00644 & 112 & 112 & 0 \\
\hline S-box 10 & 0.48438 & 0.50739 & 0.00981 & 112 & 112 & 0 \\
\hline S-box 11 & 0.47656 & 0.49784 & 0.00950 & 112 & 112 & 0 \\
\hline S-box 12 & 0.47070 & 0.49679 & 0.01026 & 112 & 112 & 0 \\
\hline S-box 13 & 0.48242 & 0.50021 & 0.01085 & 112 & 112 & 0 \\
\hline S-box 14 & 0.49023 & 0.50718 & 0.00901 & 112 & 112 & 0 \\
\hline S-box 15 & 0.48438 & 0.50265 & 0.00883 & 112 & 112 & 0 \\
\hline S-box 16 & 0.48633 & 0.50544 & 0.00861 & 112 & 112 & 0 \\
\hline
\end{tabular}

TABLE 21: Linear and differential approximation probability analysis.

\begin{tabular}{|c|c|c|c|c|}
\hline \multirow{2}{*}{ S-box } & \multicolumn{2}{|c|}{ Linear approximation probability } & \multicolumn{2}{|c|}{ Differential approximation probability } \\
\hline & Max count & LP & Max value & $\mathrm{DP}$ \\
\hline S-box 1 & 144 & 0.0625 & 4 & 0.015625 \\
\hline S-box 1 & 144 & 0.0625 & 4 & 0.015625 \\
\hline S-box 2 & 144 & 0.0625 & 4 & 0.015625 \\
\hline S-box 3 & 144 & 0.0625 & 4 & 0.015625 \\
\hline S-box 4 & 144 & 0.0625 & 4 & 0.015625 \\
\hline S-box 5 & 144 & 0.0625 & 4 & 0.015625 \\
\hline S-box 6 & 144 & 0.0625 & 4 & 0.015625 \\
\hline S-box 7 & 145 & 0.0664 & 4 & 0.015625 \\
\hline S-box 8 & 144 & 0.0625 & 4 & 0.015625 \\
\hline S-box 9 & 144 & 0.0625 & 4 & 0.015625 \\
\hline S-box 10 & 144 & 0.0625 & 4 & 0.015625 \\
\hline S-box 11 & 144 & 0.0625 & 4 & 0.015625 \\
\hline S-box 12 & 144 & 0.0625 & 4 & 0.015625 \\
\hline S-box 13 & 144 & 0.0625 & 4 & 0.015625 \\
\hline S-box 14 & 144 & 0.0625 & 4 & 0.015625 \\
\hline S-box 15 & 144 & 0.0625 & 4 & 0.015625 \\
\hline S-box 16 & 144 & 0.0625 & 4 & 0.015625 \\
\hline
\end{tabular}


TABLE 22: Comparison of the performance indexes of the proposed S-boxes with some standard S-boxes.

\begin{tabular}{|c|c|c|c|c|c|c|}
\hline $8 \times 8$ S-boxes & Nonlinearity & SAC & $\mathrm{BIC}$ & BIC-SAC & DP & LP \\
\hline AES S-box & 112 & 0.5058 & 112 & 0.504 & 0.0156 & 0.062 \\
\hline APA S-box & 112 & 0.4987 & 112 & 0.499 & 0.0156 & 0.062 \\
\hline Gray S-box & 112 & 0.5058 & 112 & 0.502 & 0.0156 & 0.062 \\
\hline Skipjack S-box & 105.7 & 0.4980 & 104.1 & 0.499 & 0.0468 & 0.109 \\
\hline Xyi S-box & 105 & 0.5048 & 103.7 & 0.503 & 0.0468 & 0.156 \\
\hline Residue prime & 99.5 & 0.5012 & 101.7 & 0.502 & 0.2810 & 0.132 \\
\hline Reference [27] & 106 & 0.4978 & 103.92 & - & - & - \\
\hline Reference [28] & & 0.505 & - & - & - & - \\
\hline Reference [29] & 104 & 0.5241 & 103 & 0.50181 & 0.1625 & 0.0486 \\
\hline S-box 1 & 112 & 0.496094 & 112 & 0.50119 & 0.015625 & 0.0625 \\
\hline S-box 2 & 112 & 0.495117 & 112 & 0.50600 & 0.015625 & 0.0625 \\
\hline S-box 3 & 112 & 0.494141 & 112 & 0.50202 & 0.015625 & 0.0625 \\
\hline S-box 4 & 112 & 0.50708 & 112 & 0.50656 & 0.015625 & 0.0625 \\
\hline S-box 5 & 112 & 0.503174 & 112 & 0.50105 & 0.015625 & 0.0625 \\
\hline S-box 6 & 112 & 0.501709 & 112 & 0.49819 & 0.015625 & 0.0625 \\
\hline S-box 7 & 112 & 0.502441 & 112 & 0.50593 & 0.015625 & 0.0625 \\
\hline S-box 8 & 112 & 0.503906 & 112 & 0.50251 & 0.015625 & 0.0664 \\
\hline S-box 9 & 112 & 0.485596 & 112 & 0.50258 & 0.015625 & 0.0625 \\
\hline S-box 10 & 112 & 0.509766 & 112 & 0.50739 & 0.015625 & 0.0625 \\
\hline S-box 11 & 112 & 0.50415 & 112 & 0.49784 & 0.015625 & 0.0625 \\
\hline S-box 12 & 112 & 0.501221 & 112 & 0.49679 & 0.015625 & 0.0625 \\
\hline S-box 13 & 112 & 0.500977 & 112 & 0.50021 & 0.015625 & 0.0625 \\
\hline S-box 14 & 112 & 0.508301 & 112 & 0.50718 & 0.015625 & 0.0625 \\
\hline S-box 15 & 112 & 0.498779 & 112 & 0.50265 & 0.015625 & 0.0625 \\
\hline S-box 16 & 112 & 0.496582 & 112 & 0.50544 & 0.015625 & 0.0625 \\
\hline
\end{tabular}

TABLE 23: MLC analyses for the proposed S-boxes.

\begin{tabular}{|c|c|c|c|c|c|}
\hline S-boxes & Entropy & Correlation & Contrast & Energy & Homogeneity \\
\hline S-box 1 & 7.2763 & 0.1032 & 8.8505 & 0.0175 & 0.4499 \\
\hline S-box 2 & 7.2763 & 0.1246 & 9.1517 & 0.0174 & 0.4530 \\
\hline S-box 3 & 7.2763 & 0.0895 & 9.5748 & 0.0175 & 0.4532 \\
\hline S-box 4 & 7.2763 & 0.1486 & 8.9132 & 0.0184 & 0.4579 \\
\hline S-box 5 & 7.2763 & 0.1277 & 9.2077 & 0.0195 & 0.4714 \\
\hline S-box 6 & 7.2763 & 0.1326 & 9.8130 & 0.0178 & 0.4532 \\
\hline S-box 7 & 7.2642 & 0.0904 & 10.0599 & 0.0177 & 0.4438 \\
\hline S-box 8 & 7.2763 & 0.1256 & 9.6635 & 0.0182 & 0.4509 \\
\hline S-box 9 & 7.2763 & 0.1099 & 8.9653 & 0.0179 & 0.4609 \\
\hline S-box 10 & 7.2763 & 0.0747 & 9.7106 & 0.0177 & 0.4453 \\
\hline S-box 11 & 7.2763 & 0.0927 & 9.7905 & 0.0182 & 0.4529 \\
\hline S-box 12 & 7.2763 & 0.0879 & 9.8720 & 0.0182 & 0.4531 \\
\hline S-box 13 & 7.2763 & 0.0868 & 8.5569 & 0.0188 & 0.4606 \\
\hline S-box 14 & 7.2763 & 0.0862 & 8.6308 & 0.0179 & 0.4510 \\
\hline S-box 15 & 7.2763 & 0.1245 & 9.3829 & 0.0184 & 0.4522 \\
\hline S-box 16 & 7.2763 & 0.0843 & 9.5898 & 0.0179 & 0.4533 \\
\hline
\end{tabular}

the minimum value of SAC is 0.453125 for the first 10 $\mathrm{S}$-boxes including $12^{\text {th }}$ and $14^{\text {th }} \mathrm{S}$-boxes. The average value of SAC lies in the interval [0.4856, 0.509766].

3.3. Bit Independent Criterion. Another algebraic criterion (BIC) is used to evaluate the strength of S-box, which is presented by Detombe and Tavares in [26]. In Table 14, the outcomes of BIC to SAC and BIC for the proposed S-boxes are given. The minimum BIC to SAC value is 0.47070 for $12^{\text {th }}$ S-box and the highest minimum value is 0.49219 for $2^{\text {nd }}$ S-box. The average BIC to SAC lies between 0.49679 and 0.50739. Similarly, the square deviation values for all the proposed S-boxes are given in Table 20. The maximum and average value of BIC is 112 for all S-boxes. It is depicted that the proposed S-boxes give the nearest best value of BIC analyses.

3.4. Linear Approximation Probability. Matsui defines the extreme value of the imbalance of an event as the linear approximation probability. It is notable that the parity of the input bits that is, the mask $G_{l}$, is equal to the parity of the output bits, i.e., the mask $G_{m}$. The linear approximation probability of a given S-box is defined in the following equation: 


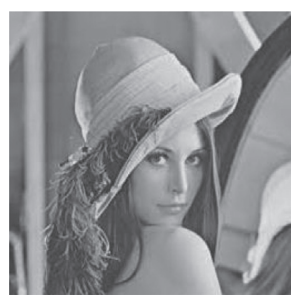

(a)

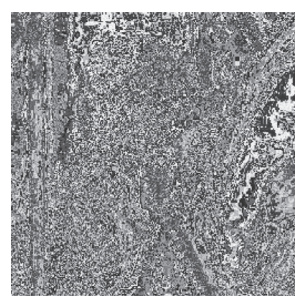

(f)

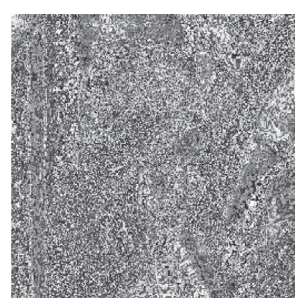

(k)

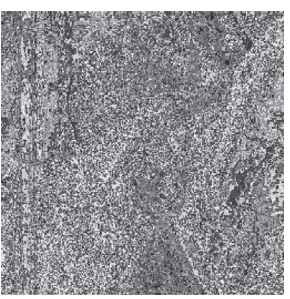

(b)

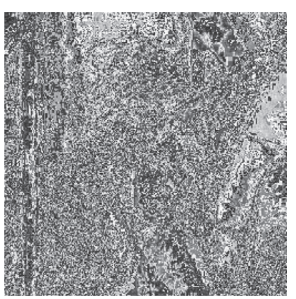

(g)

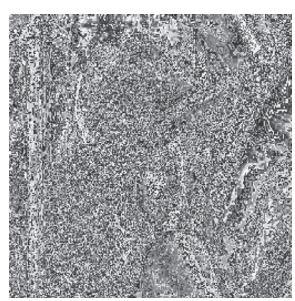

(1)

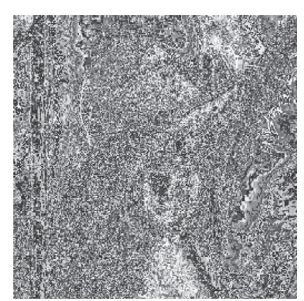

(c)

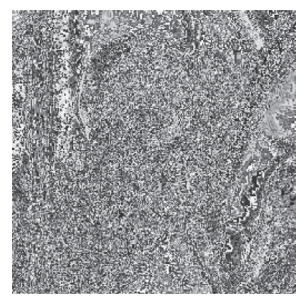

(h)

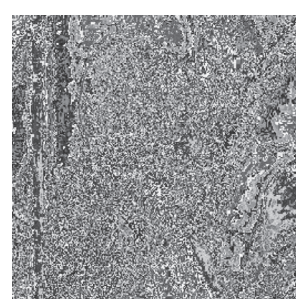

(m)

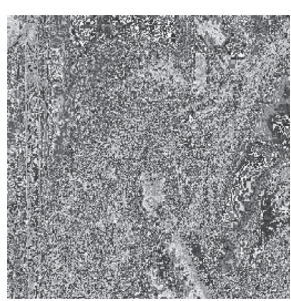

(d)

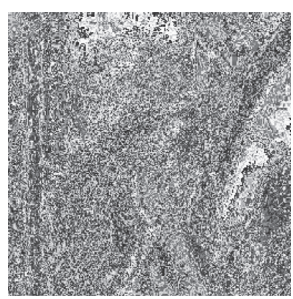

(i)

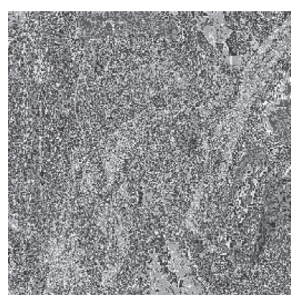

(n)

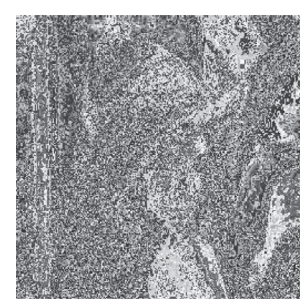

(e)

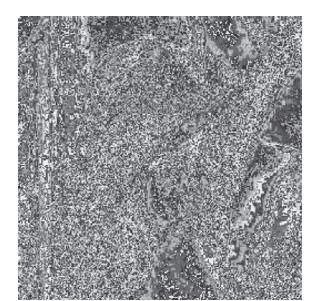

(j)

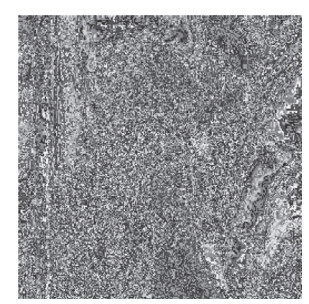

(o)

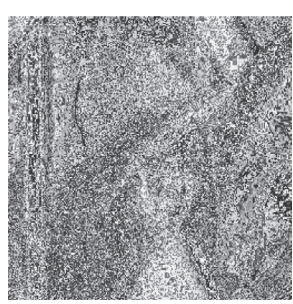

(p)

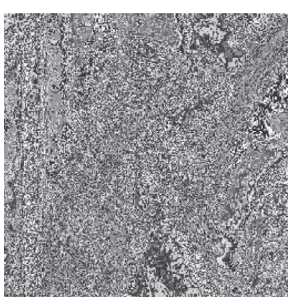

(q)

Figure 2: Original Lena image and the encrypted images using all 16 primitive irreducible polynomials S-boxes. (a) Lena image, (b) S-box 1, (c) S-box 2, (d) S-box 3, (e) S-box 4, (f) S-box 5, (g) S-box 6, (h) S-box 7, (i) S-box 8, (j) S-box 9, (k) S-box 10, (l) S-box 11, (m) S-box 12, (n) Sbox 13, (o) S-box 14, (p) S-box 15, and (q) S-box 16.

$$
\mathrm{LP}=\max _{G_{l}, G_{m} \neq 0}\left|\frac{\#\left\{l \in X \mid l \cdot G_{l}=S(l) \cdot G_{m}\right\}}{2^{n}}-\frac{1}{2}\right|,
$$

where $G_{l}$ and $G_{m}$ are input and output masks, respectively, and the set " $X$ " represents the set of all possible inputs; $2^{n}$ is the number of elements of $X$. The value of linear approximation indicates the strength of S-box against various linear attacks. In Table 21, the maximum count and the LP value for all proposed S-boxes is 144 and 0.0625 . These values of LP of the proposed S-boxes are appropriate against linear attacks.

3.5. Differential Approximation Probability. The degree of differential uniformity is known as differential approximation probability $\left(\mathrm{DP}^{\mathrm{s}}\right)$ of S-box. Mathematically, it can be given as

$$
\mathrm{DP}^{s}(\Delta l \longrightarrow \Delta m)=\left[\frac{\#\{l \in X \mid S(l) \pm S(l \pm \Delta l)=\Delta m\}}{2^{m}}\right]
$$

Briefly, it can be explained as follows: an input differential $\Delta l_{i}$ must be mapped to an output differential $\Delta m_{i}$ uniquely for each $i$. Here, $X$ represents all the possible input values and the number of its elements is given by $2^{m}$. Table 21 depicts the results of DP, which include the maximum and DP value.

Moreover, Table 22 represents the values of proposed S-boxes along with AES, Skipjack, Xyi, APA, Gray, and residue prime $\mathrm{S}$-boxes.

3.6. Statistical Analyses. To evaluate the visual strength of the substitution with the help of the proposed S-boxes, various statistical analyses are made on the host 
and substituted images. In this proposed work, statistical analyses like homogeneity, entropy, contrast, energy, and correlation are used to evaluate the substitution ability of the 16 proposed S-boxes. These analyses are given as

$$
\begin{aligned}
\text { correlation } & =\sum_{k, l} \frac{(k-\mu k)(l-\mu l)}{\sigma_{k} \sigma_{l}} p(k, l) \\
\text { contrast } & =\sum_{k, l}|k-l|^{2} p(k, l) \\
\text { entropy } & =-\sum_{k, l} \operatorname{pr}(p(k, l)) \log \operatorname{Pr}(p(k, l)) \\
\text { homogeneity } & =\sum_{k, l} \frac{p(k, l)}{1+|k-l|}, \\
\text { energy } & =-\sum_{k, l} p(k, l)^{2}
\end{aligned}
$$

where $k, l$ give the row and column locations of an image. The pixel value at $k^{\text {th }}$ row and $l^{\text {th }}$ column is represented by $p(k, l)$ and $\operatorname{Pr}(p(k, l))$ is the probability of the image pixel. In equation (8), $\mu$ and $\sigma$ are mean and standard deviation, respectively.

Correlation analysis helps to find the similarity between the host and substituted image. The correlation analysis provides the range which indicates the perfect, negative, and positive correlation. This is $[-1,1]$ interval for correlation and value of 1 indicates the perfect correlation.

The randomness of the digital image can be calculated with the help of entropy. The higher value of entropy from the interval $[0,8]$ represents the higher amount of randomness in a digital image. For any viewer, it is only possible with the help of contrast analysis to intensely recognize the objects in the texture of an image. With the help of contrast analyses, one can observe the maximum distinction in image pixels. The range of the contrast can be given by $\left[(\operatorname{size}(\text { Image })-1)^{2}\right]$. For constant image, the value of contrast is zero. The goal of finding close distribution between the matrix and its diagonal is obtained in homogeneity analysis. The matrix used in this analysis is named gray level cooccurrence matrix (GLCM) and the range of homogeneity lies between 0 and 1 . The range for energy analysis also lies in the interval $[0,1]$. The results of Table 23 are obtained by applying these analyses on the original and encrypted images. For all the proposed 16 S-boxes, we calculated the values of the statistical analyses.

A $256 \times 256$ JPEG image of Lena is considered for MLC analysis. Figure 2 shows the results of image encryption with 16 proposed S-boxes.

\section{Balanced Boolean Function}

4.1. Balance Property. The imbalance of a Boolean function weak system against linear cryptanalysis highlights the importance of balance property. The balance property indicates that the higher the magnitude of a function's imbalance, the more the chances of a high probability linear approximation. A Boolean function $f: \mathbb{Z}_{2}^{n} \longrightarrow \mathbb{Z}_{2}$ is balanced. If the cardinality or Hamming weight of these two functions, that is, $\{x: f(x)=0\}$ and $\{x: f(x)=1\}$ is the same, then it is named the balance function.

4.2. Balance Property of the Proposed S-Box. All the Boolean functions $f_{i}, i=1,2,3, \ldots, 8$ involved in proposed S-boxes are balanced just like the Boolean functions of AES, $S_{8}$, AES and other well-known S-boxes. The nonlinearity of the proposed S-boxes is equal to 112 .

\section{Conclusion}

In this paper, a scheme for the synthesis of $8 \times 8 \mathrm{~S}$-boxes over 16 isomorphic Galois fields is presented. Here, we fixed all the parameters of affine power affine transformation, that is, $a, b, c, d$ for 16 S-boxes. We have 16 primitive irreducible polynomials of degree 8 and they prompt us to construct 16 Galois field extensions of order 256. By using elements of the Galois field, corresponding to each different pair of the parameters, one can construct different S-boxes. These S-boxes obtained as a result of APA transformation which is bijective, pass nonlinearity test, and out bit independent criterion (BIC) which demonstrates that the existing S-boxes have high confusion producing capability. The evaluation of constructed S-boxes is done with some algebraic and statistical analyses. The results of these analyses highlight the characteristics of all the proposed S-boxes and later these S-boxes are equated with some of the existing S-boxes. In addition to this, we also ensured that all these constructed S-boxes are balanced that guarantee the strength of our S-boxes. Hence, we have concluded that a large class of S-boxes can be obtained by varying parameters of affine power affine transformations. These S-boxes can be used for secure communication.

\section{Data Availability}

The data that support the findings of this study are available from the corresponding author upon reasonable request.

\section{Conflicts of Interest}

There are no conflicts of interest among the authors.

\section{Acknowledgments}

The authors extend their appreciation to the Deanship of Scientific Research at King Khalid University for funding this work through research groups program under Grant no. R.G.P. 1/234/41.

\section{References}

[1] J. Daemen and V. Rijmen, “The design of Rijndael: AES,"in The Advanced Encryption Standard, Springer, Berlin, Germany,2002.

[2] R. Zimmermann, A. Curiger, H. Bonnenberg, H. Kaeslin, N. Felber, and W. Fichtner, "A $177 \mathrm{Mb} / \mathrm{s}$ VLSI implementation of the international data encryption algorithm," 
IEEE Journal of Solid-State Circuits, vol. 29, no. 3, pp. 303307, 1994.

[3] E. Biham and A. Shamir, "Differential cryptanalysis of DESlike cryptosystems," Journal of Cryptology, vol. 4, no. 1, pp. 3-72, 1991.

[4] National Bureau of Standards, Data Encryption Standard, Vol. 46, FIPS Publication, U.S. Department of Commerce, Washington, DC, USA, 1977.

[5] L. Cui and Y. Cao, "A new S-box structure named affinepower-affine," International Journal of Innovative Computing, Information and Control, vol. 3, no. 3, pp. 751-759, 2007.

[6] I. Hussain, T. Shah, H. Mahmood, and M. A. Gondal, "Construction of S8 Liu J S-boxes and their applications," Computers \& Mathematics with Applications, vol. 64, no. 8, pp. 2450-2458, 2012.

[7] M. T. Tran, D. K. Bui, and A. D. Duong, "Gray S-box for advanced encryption standard,"in Proceedings of the 2008 International Conference on Computational Intelligence and Security, vol. 1, IEEE, Suzhou, China, December 2008.

[8] T. Shah and D. Shah, "Construction of highly nonlinear S-boxes for degree 8 primitive irreducible polynomials over $\mathbb{Z} 2$," Multimedia Tools and Applications, vol. 78, no. 2, pp. 1219-1234, 2019.

[9] M. Khan, T. Shah, H. Mahmood, M. A. Gondal, and I. Hussain, "A novel technique for the construction of strong S-boxes based on chaotic Lorenz systems," Nonlinear Dynamics, vol. 70, no. 3, pp. 2303-2311, 2012.

[10] M. Khan and T. Shah, "An efficient construction of substitution box with fractional chaotic system," Signal, Image and Video Processing, vol. 9, no. 6, pp. 1335-1338, 2015.

[11] I. Hussain, T. Shah, H. Mahmood, and M. A. Gondal, "A projective general linear group based algorithm for the construction of substitution box for block ciphers," Neural Computing and Applications, vol. 22, no. 6, pp. 1085-1093, 2013.

[12] Y. Tian and Z. Lu, "Novel permutation-diffusion image encryption algorithm with chaotic dynamic S-box and DNA sequence operation," AIP Advances, vol. 7, no. 8, Article ID 085008, 2017.

[13] M. Khan, F. Masood, A. Alghafis, M. Amin, and S. I. Batool Naqvi, "A novel image encryption technique using hybrid method of discrete dynamical chaotic maps and Brownian motion," PLoS One, vol. 14, no. 12, Article ID e0225031, 2019.

[14] M. Khan and T. Shah, "A novel cryptosystem based on general linear group," 3D Research, vol. 6, no. 1, 2015.

[15] D. Shah, T. Shah, and S. S. Jamal, "A novel efficient image encryption algorithm based on affine transformation combine with linear fractional transformation," Multidimensional Systems and Signal Processing, vol. 31, no. 3, pp. 885-905, 2020.

[16] Y. Naseer, D. Shah, and T. Shah, "A novel approach to improve multimedia security utilizing 3D mixed chaotic map," Microprocessors and Microsystems, vol. 65, pp. 1-6, 2019.

[17] K. E. A. Skipjack, “Algorithm," Specifications Version, vol. 2, no. 29, pp. 1-23, 1998.

[18] E. S. Abuelyman and A.-A. Sultan Alsehibani, "An optimized implementation of the S-box using residue of prime numbers," International Journal of Computer Science and Network Security, vol. 8, no. 4, pp. 304-309, 2008.

[19] S. Mahmood et al., "To study the effect of the generating polynomial on the quality of nonlinear components in block ciphers." Security and Communication Networks, vol. 2018, Article ID 5823230, 8 pages, 2018.
[20] M. Matsui, "Linear cryptanalysis method for DES cipher," in Advances in Cryptology-Eurocrypt'93, pp. 386-397, Springer Berlin Heidelberg, Heidelberg, Germany, 1993.

[21] Y. Wang, Q. Xie, Y. Wu, and B. Du, "A software for S-box performance analysis and test," in Proceedings of the 2009 International Conference on Electronic Commerce and Business Intelligence, pp. 125-128, IEEE, Beijing, China, June 2009.

[22] M. A. Gondal, A. Raheem, and I. Hussain, "A scheme for obtaining secure S-boxes based on chaotic Baker's map," $3 D$ Research, vol. 5, no. 3, pp. 5-17, 2014.

[23] A. Belazi, R. Rhouma, and S. Belghith, "A novel approach to construct S-box based on Rossler system," in Proceedings of the 2015 International Wireless Communications and Mobile Computing Conference (IWCMC), pp. 611-615, Dubrovnik, Croatia, August 2015.

[24] A. F. Webster and S. E. Tavares, "On the design of S-boxes," in Advances in Cryptology-Crypto'85 Proceedings, pp. 523-534, Springer Berlin Heidelberg, Heidelberg, Germany, 1985.

[25] F. Sattar and M. Mufti, "Spectral characterization and analysis of avalanche in cryptographic substitution boxes using walshhadamard transformations," International Journal of Computer Applications, vol. 28, no. 6, 2011.

[26] J. Detombe and S. Tavares, "On the design of S-boxes," Advances in Cryptology: Proceedings of CRYPTO_92, Springer Berlin Heidelberg, Heidelberg, Germany, Lecture Notes in Computer Science, 1992.

[27] A. K. Farhan, R. S. Ali, H. Natiq, and N. M. G. Al-Saidi, "A new S-box generation algorithm based on multistability behavior of a plasma perturbation model," IEEE Access, vol. 7, pp. 124914-124924, 2019.

[28] A. Farhan, R. Subhi, H. Rashed Yassein, and N. Al-Saidi, “A new approach to generate multi S-boxes based on RNA computing," International Journal of Innovative Computing, Information and Control: IJICIC, vol. 16, no. 1, pp. 331-348, 2020.

[29] D. Shah and T. Shah, "Binary galois field extensions dependent multimedia data security scheme," Microprocessors and Microsystems, vol. 77, Article ID 103181, 2020. 